\title{
SOBRE EL CONCEPTO DE OBJETO EN
}

EL "TRACTATUS"

RAFAEL BURGOS

Universidad de los Andes,

Mérida, Venezuela

Wo unsere Sprache uns einen Körper vermuten lässt, und kein Körper ist, dort, möchten wir sagen, sei ein Geist.

L. WITTGENSTEIN

Según el Tractatus el mundo está constituido por la totalidad de los hechos $(1 ; 1.1 ; 1.11)$. Entre los hechos que constituyen el mundo algunos tienen un 'status' peculiar: son producidos o utilizados para representar o reproducir otros hechos $(2.1 ; 2.141)$. Entre los hechos que pueden cumplir esta función y que son llamados, en general, imágenes, el Tractatus presta especial atención a los hechos lingüisticos. Llamamos hechos lingüsticos a las proposiciones, imágenes de la realidad (4.01). El lenguaje está constituido por la totalidad de las proposiciones (4.001). El lenguaje es, pues, un conjunto de hechos utilizados para reproducir otros hechos. Se distinguen dos tipos de proposiciones: proposiciones elementales y proposiciones no-elementales. Las proposiciones no-elementales son funciones de las proposiciones elementales $(4.4 ; 4.411 ; 5 ; 5.3)$.

Las proposiciones no-elementales son unívocamente analizables en función de proposiciones elementales (3.25; 4.221). Dadas todas las proposiciones elementales, se pueden construir todas las proposiciones no-elementales $(4.51 ; 4.52)$. Las proposiciones elementales son así los hechos lingüísticos 
primarios y sobre ellas se construyen los demás hechos lingüísticos. Las proposiciones elementales se refieren a hechos atómicos (4.21). Los hechos atómicos son configuraciones de objetos ${ }^{1}(2.01 ; 2.0272)$ y las proposiciones elementales son configuraciones de nombres $(3.202 ; 3.21 ; 3.22)$. Las proposiciones elementales reproducen los hechos atómicos. Para ello, los nombres en la proposición elemental corresponden a los objetos en el hecho atómico y las relaciones de los nombres en la proposición elemental corresponden a las relaciones de los objetos en el hecho atómico (2.13; $2.131 ; 2.15)$. Los objetos, configurados en hechos atómicos, vienen a ser de esta manera la referencia última del lenguaje.

Por otra parte, los objetos forman la sustancia del mundo (2.021). Ellos son lo inalterable y subsistente (2.024; $2.027 ; 2.0271$ ), mientras sus configuraciones son cambiantes e inestables $(2.0271 ; 2.0272)$.

Cuando el Tractatus habla de hechos, utiliza por lo menos cinco términos, tres de los cuales son de especial importancia; hecho (Tatsache), hecho atómico (Sachverhalt) y estado de cosas (Sachlage). ${ }^{2}$ Los significados y diferencias de estos términos no han podido ser establecidos definitivamente, ${ }^{3}$ pero nos parece lícito decir, en general, que los hechos son, mediata o inmediatamente, posible o realmente, configuraciones de objetos. Podremos, por tanto, decir de todos ellos. que son cambiantes e inestables.

Los objetos son así los elementos ontológicos fundamentales, a la vez que la referencia última del lenguaje. De allí su enorme importancia. Un estudio del Tractatus que tenga

1 Al hablar del término 'objeto' del Tractatus, y siempre que no se diga lo contrario, nos estaremos refiriendo tanto al término 'Gegenstand' como a sus sinónimos 'Sache' y 'Ding'. La sinonimia es establecida en 2.01 “Der Sachverhalt is eine Verbindung von Gegenständen. (Sachen, Dingen)" $Y$ no sabemos de ningún autor que no la reconozca.

2 Otros dos son 'Factum' y 'Ereignis'.

${ }^{3}$ Cf., v. gr., Anscombe, An Introduction to Wittgenstein's Tractatus (London, 1959), p. 30, passim; Stenius, Wittgenstein's Tractatus (Oxford, 1964), p. 29 ss, passim; Black. A Campanion to Wittgenstein's Tractatus (Cambridge 1964), p. 41 ss, passim; Griffin. Wittgenstein's Logical Atomism (London, 1965) p. $30 \mathrm{ss,}$, passim. 
que ver con la cuestión del lenguaje o con sus ramificaciones - cuestiones que constituyen la mayor parte de la obra, si no la más importante- no puede prescindir de aclarar satisfactoriamente el concepto de objeto.

Específicamente nos proponemos investigar dos cuestiones:

1) ¿Cuál es la ubicación sistemática del concepto de objeto en el Tractatus? Queremos determinar a este respecto si el concepto de objeto tiene o no carácter de supuesto dentro del sistema expuesto en la obra; y

2) ¿Qué interpretación se debe dar al concepto de objeto?

\section{II}

Nuestro primer problema, el de la ubicación sistemática del concepto de objeto, puede ser atacado determinando y analizando los pasajes en que se plantee la necesidad del concepto dentro del sistema. El único pasaje donde esta cuestión es tratada directamente es:

2.021 Die Gegenstände bilden die Substanz der Welt. Darum können sie nicht zusammengesetzt sein.

2.0211 Hätte die Welt keine Substanz, so würde, ob ein Satz Sinn hat, davon abhängen, ob ein anderer Satz wahr ist.

2.0212 Es wäre dann unmöglich, ein Bild der Welt (wahr oder falsch) zu entwerfen.

Pasaje que podemos glosar de la siguiente manera: una proposición puede ser verdadera o falsa $(2.21 ; 4.06)$. Salvo en el caso de las proposiciones lógicas, cuya verdad o falsedad puede ser reconocida en base sólo al signo proposicional (6.113), la verdad o falsedad de una proposición no puede ser determinada independientemente de la realidad a la cual se refiere $(2.223 ; 2.224 ; 2.225 ; 4.06)$. Las proposiciones no-elementales son funciones de verdad de las pro. posiciones elementales $(4.4 ; 4.41 ; 4.31 ; 5 ; 5.01 ; 5.3 ; 5.31$; 
5.32), mientras que las proposiciones elementales son funciones de verdad de sí mismas. Ahora bien, el sentido de una proposición puede ser comprendido independientemente de su verdad o falsedad $(2.22 ; 4.024 ; 4.061)$. Por el contrario, el valor de verdad de una proposición depende para su determinación del sentido de ésta (2.222). Por otra parte, el sentido de una función de verdad de una proposición dada es una función del sentido de esta proposición (5.2341). Luego, el sentido de las proposiciones no-elementales es una función del sentido de las proposiciones elementales. Si negáramos la existencia de objetos, esto es, de elementos ontológicos simples a cuyas configuraciones se refieren las proposiciones elementales, éstas perderían su carácter de elementales - ya que las proposiciones sobre complejos deben ser analizadas en proposiciones sobre sus componentes $(2.0201 ; 3.24)$ - y tanto su sentido como su valor de verdad quedarían referidos a nuevas proposiciones, etc. De esta manera el sentido de una proposición cualquiera se pierde en una regresión 'ad infinitum'. Pero como se da por sentado que hay proposiciones con sentido, se hace necesario afirmar la existencia de objetos. En realidad el pasaje presenta la regresión en una forma ligeramente diferente, a saber: hemos visto que el valor de verdad de una proposición depende de su sentido; entonces, decir que el sentido de una proposición depende del valor de verdad de otra equivale a establecer la regresión, pues, a su vez, el sentido de la nueva proposición dependerá del valor de verdad de otra, sin poder detener el análisis, pues para ello habría que encontrar una proposición cuyo valor de verdad dependiera de su sentido, lo cual iría contra el enunciado.

Nuestro pasaje tiene la forma de una 'reductio ad absurdum'. Presentando en su forma más simple rezaría: las proposiciones se clasifican en elementales y no-elementales. El sentido de las proposiciones no-elementales depende del sentido de las proposiciones elementales y el sentido de éstas depende de la existencia de entidades extra-lingüísticas sim- 
ples (objetos). Luego, el sentido de cualquier proposición depende, en última instancia, de la existencia de objetos. Si negamos la existencia de objetos, tendremos que negar la existencia de proposiciones con sentido. Dado que existen proposiciones con sentido, habrá que afirmar la existencia de objetos.

Si nuestra glosa es correcta, la existencia de objetos es un supuesto, pues no se podría hablar de demostración en el pasaje estudiado sin considerar falaz tal demostración.

\section{III}

En cuanto a nuestro segundo problema, la interpretación del concepto de objeto, tendremos que empezar por discutir la posibilidad y alcance de tal interpretación, pues entre los comentaristas del Tractatus se observan dos tendencias: una que propugna la tesis de que el lenguaje de que se habla en la obra es, en última instancia, el lenguaje corriente, entendiendo por lenguaje corriente cualquier lenguaje históricamente dado; y otra tendencia que defiende la tesis de que el lenguaje de que habla el Tractatus no es ningún lenguaje históricamente dado, sino un lenguaje ideal al cual tiende o debe tender todo lenguaje dado. ${ }^{4}$ Los defensores de la segunda tesis llegan a la conclusión de que ciertos conceptos, como los de objeto, nombre, hecho atómico, proposición elemental, etc., son meras inferencias en el desarrollo de la teoría del lenguaje ideal y que, por lo tanto, no necesitan

4 Sobre el problema de los diferentes 'lenguajes' del Tractatus nos parece muy útil el artículo de Richard Bernstein, con el cual estamos de acuerdo en líneas generales. Bernstein distingue tres 'lenguajes' en la obra: "...the perspicuous language ordinary Language and the Ladder Language. The perspicuous language is an aid for understanding how language works when we use it to make true and false statements. It is not an ideal language which ordinary language must 'approach' in order to fulfil its function. To describe this perspicuous language, we ... use ... the ladder language which must not be confused with the object language that it describes" Bernstein, "Wittgenstein's Three Lenguages" (in: Copi, Essays on Wittgen. stein's Tractatus, London, 1966), p. 236-237. 
ser interpretados fuera de la teoria, ni mucho menos ejem. plificados. Uno de los representantes más radicales de esta tesis es Max Black y el texto más importante en que se refiere al problema es:

"His philosophical semantic is that of a lingua abscon. dita grounded in 'elementary propositions' whose existence is guaranteed only by metaphysical inference. We can produce no elementary propositions and would not recongnize them if we had them. Consequently, we can form no clear idea of what genuine 'names', the constituents of elementary propositions, are like, no any clear conception of the nature and logical form of the objects for which these names stand. (The arguments in which some writers have engaged about whether objects are to be counted as particulars or universals, whether elementary propositions are relational or not, and so on, are bound to be futile."

Ahora bien, según el Tractatus, las proposiciones no elementales son funciones de verdad de las proposiciones elementales y el sentido de una proposición no-elemental es función del sentido de las proposiciones elementales en las cuales se analiza. Las proposiciones no-elementales de que habla Wittgenstein son las del lenguaje corriente, ya sea en su forma habitual, ya simbolizadas de tal manera que sus estructuras y relaciones resulten más claras, y no las de una 'lingua abscondita'; para demostrarlo bastan 3.141; 3.143 ; 3.1431; 3.323; 4.001; 4.0031 ; 4.011 ; 4.016 ; 4.025; $4.026 ; 4.11 ; 4.1272 ; 5.02 ; 5.515 ; 5.521 ; 5.5302 ; 5.541$; $5.5563 ; 6.1232 ; 6.31 ; 6.34 ; 6.341 ; 6.343$. Si las proposiciones elementales fueran incognoscibles: o el sentido y valor de verdad de las proposiciones no elementales, esto es, de las proposiciones del lenguaje corriente, podrían ser determinados sin referencia a ellas, lo cual contradice la tesis wittgensteiniana o, en el mejor de los casos, la hace superflua; o el sentido y el valor de verdad de las proposiciones

5 Black, op. cit., p. 11. 
no-elementales no podrían ser determinados en absoluto. En otras palabras, o las proposiciones elementales - y con ellas los nombres-, los objetos y los hechos atómicos son cognoscibles, o la teoría que las propone resulta incoherente, o el lenguaje no puede comunicar sentido alguno.

\section{IV}

Alexander Maslow dice contar en el Tractatus no menos de una docena de posibles interpretaciones del término 'objeto' pero sólo enuncia y se ocupa de cuatro: objetos sensibles, cosas (en el sentido corriente del término), entidades ontológicas y 'designata'. El mismo Maslow encuentra las tres primeras interpretaciones impracticables y ofrece la última como la más razonable desde un punto de vista positivista, aunque su conclusión general respecto al concepto de objeto es que Wittgenstein no tenía, para la época en que escribió el Tractatus, una idea clara de lo que quería decir con 'objeto'.

Prescindiendo de comentar los principios hermenéuticos de Maslow, nos ocuparemos de las interpretaciones que da de los objetos del Tractatus como cosas, entidades ontológicas y 'designata', dejando la interpretación como datos senso-perceptibles para ser tratada más adelante.

El término objeto puede ser considerado como referido a cosas, en el sentido corriente del término, en

3.1431 Sehr klar wird das Wesen des Satzzeichens, wenn wir es uns, statt aus Schriftzeichen, aus räumlichen Gegenständen (etwa Tischen, Stühlen, Büchern) zusamgesetzt denken.

y, si la expresión 'objeto espacial' fuera a ser entendida siempre en este sentido, en 2.0121 y 2.0131 . Pero si bien toda cosa, en el sentido en que se llama cosa a una silla

- Maslow, A Study in Wittgenstein's Tractatus (Berkeley, 1961), pp. 5, 8, passim. 
o una mesa, es espacial, no todo objeto espacial tiene que ser una cosa en el mismo sentido.

Ahora bien, de los objetos se dice en el Tractatus que son simples $(2.02 ; 2.021)$. Y ya esto bastaría para descartar las cosas, en el sentido corriente, de las interpretaciones del término 'objeto', pues tales cosas no son simples en ningún sentido. Pero además se dice en la obra que los objetos son lo inalterable y subsistente $(2.024 ; 2.027$; 2.0271 ), características que en modo alguno corresponden a las cosa de la vida diaria.

Otro uso del término rechazable por las mismas razones es el de 5.02.

Maslow pretende que Wittgenstein usa a veces el término 'objeto' para referirse a las entidades ontológicas últimas, a partir de las cuales se constituye el mundo en sí. $\mathrm{Si}$ por 'mundo en si' ha de entenderse 'mundo con total independencia del conocimiento que se tenga de él', en el Tractatus ni se plantea el problema ni se habla en ninguna parte de mundo en sí; y si por el contrario, se identifican los límites del mundo con los del lenguaje (4.26; 5.5561; $5.6 ; 5.62$ ). Además, en el prólogo (p. 26) del Tractatus se dice que

"Das Buch will also dem Denken eine Grenze ziehen, oder vielmehr — nicht dem Denken sondern dem Aus. druck der Gedanken (...). Die Grenze wird also nur in der Sprache gezogen werden und was jenseits der Grenze liegt, wird einfach Unsinn sein".

Estando nuestro lenguaje construido sobre proposiciones elementales que se refieren a las configuraciones de objetos, un mundo en sí debe ser, en el Tractatus, un sinsentido.

Maslow no encuentra en el Tractatus ningún criterio satisfactorio de simplicidad, ningún criterio que permita determinar unívocamente qué se ha de entender por 'objeto', y propone resolver el problema elaborando un cri. terio para tal fin, el cual consiste en lo siguiente: 
"The simple symbols of a significant language are called names (...). The formal definition of a simple symbol is that it must have no parts which are symbols themselves (...). The mere physical appearance of the sign is accidental; what determines the simplicity of the symbol is the definition or the grammar of the symbol (...). What a name, that is, a simple symbol, refers to in the world is to be considered as simple object or element in the world. We can take any object whatsoever in our experience and consider it as an element".?

Esta posibilidad de interpretación es estudiada por Wittgenstein en las entradas 14.6.15 y 15.6.15 de Notebooks, pero no creemos que sea aplicable al Tractatus. La aplicación de tal criterio conduciría a la existencia de objetos complejos, lo cual, en primer lugar, estaría en contradicción con 2.02 y 2.021. En segundo lugar, como dice Favrholdt, ${ }^{8}$ si consideramos los complejos como objetos, algunas proposiciones elementales serian deducibles de otras proposiciones elementales cuyo valor de verdad dependería del valor de verdad de otras proposiciones, esto es, proposiciones elementales que no son proposiciones elementales.

\section{V}

Además de la interpreatción como 'designata' de Maslow, Favrholdt considera dos posibles interpretaciones del concepto de objeto, una que llama "atomismo fenomenalista" y otra que denomina "enfoque continuo-fenomenalista" (phenomenalistic continuity-view). De la segunda dice:

"It is certain ... that Wittgenstein has never thought of this view as a possibility".

7 Maslow, op. cit., pp. 38-39.

8 Cf. Favrholdt, An Interpretation and Critique of Wittgenstein's Tractatus (Copenhague, 1965), pp. 61-62. 
"My conclusion is ... that this phenomenalistic continuity-view cannot serve as an exemplification of Wittgenstein's conceptions of the world and reality either."

Por lo que, estando de acuerdo con Favrholdt, nos eximinos de considerarla.

La otra interpretación considera los objetos como datos senso-perceptibles, en base a:

2.013 Jedes Ding ist, gleichsam, in einem Raume möglicher Sachverhalte. Diesen Raum kann ich mir leer denken, nicht aber das Ding ohne den Raum.

2.0131 Der räumliche Gegenstand muss im unendlichen Raume liegen. (Der Raumpunkt ist eine Argumentstelle). Der Fleck im Gesichtsfeld muss zwar nicht rot sein, aber eine Farbe muss er haben: er hat sozusagen den Farbenraum um sich. Der Ton muss eine Höhe haben, der Gegenstand des Tatsinnes eine Härte, usw.

$\mathrm{Y}$ a pesar de que considera que Wittgenstein rechaza esta interpretación en 6.375 y 6.3751 , Favrholdt insiste en que el Tractatus conduce a una interpretación del concepto de objeto en este sentido, desarrollándola en la forma siguiente: los objetos (en el sentido corriente del término) pueden ser divididos o analizados por lo menos de dos maneras: 1) fenomenolísticamente, ${ }^{10}$ y 2) físicamente. Entendiendo por análisis fenomenalístico el que se efectúa de acuerdo a lo que aparece a los sentidos; y por análisis físico el que realiza por métodos físicos. Algo fenomenalísticamente indivisible puede ser físicamente divisible, pero no al contrario. $\mathrm{Si}$ se realiza un análisis fenomenalístico de, por

O Favrholdt, op. cit., pp. 67, 68.

10 Favrholdt utiliza indistintamente 'phenomenalistic' y 'phenomenalogical'. Nosotros usaremos sólo 'fenomenalístico' para evitar asociaciones inconvenientes. 
ejemplo, lo que un sujeto ve, se llegará a simples visuales. Y si ello fuera posible para los demás sentidos, podríamos quizás identificar tales simples senso-perceptivos con los objetos del Tractatus.

Dos argumentos, además de los que encuentra el mismo Favrholdt, parecen suficientes para demostrar que si el Tractatus conduce a una interpretación de este tipo, conduce a una interpretación contradictoria con el resto de la obra.

En primer lugar, tales simples senso-perceptivos, suponiendo que pudieran ser determinados, serían esencialmente cambiantes e inestables, contra 2.024, 2.027, 2.0271.

En segundo lugar, las proposiciones físicas que analizan tales simples, y que según 6.3431 pertenecen al lenguaje, caerían fuera del lenguaje, pues si no son funciones de verdad de proposiciones elementales, tiene que ser ellas mismas proposiciones elementales; pero no pueden serlo porque no se refieren a configuraciones de objetos (simples senso-perceptivos) y no pueden ser funciones de verdad de proposiciones elementales porque éstas respecto a ellas son proposiciones sobre complejos, esto es, no son elementales.

\section{VI}

Erik Stenius entiende bajo el término 'objeto' particulares, propiedades y relaciones. ${ }^{11}$ Esta interpretación se basa en argumentos cuya corrección es muy atacable, por decir lo menos. En primer lugar se nos dice que las cosas (particulares) sólo pueden formar parte de hechos como portadoras de predicados (propiedades y/o relaciones). Lo que debería de ser una prueba de tal afirmación es la exégesis que hace Stenius de la primera tesis $(1 ; 1.1 ; 1.2)$ del Tractatus. Ahora bien, tal exégesis se basa en la construcción de una analogía heurística para el concepto de "mundo como hecho'. El modelo analógico es el de "campo de percepción",

\footnotetext{
11 Stenius, op. cit., pp. $61-87$, passim.
} 
donde, según Stenius, queda establecido que los hechos se descomponen en particulares y predicados, los cuales constituyen categorías complementarias no reducibles unas a otras. Este análisis del campo de percepción es, sin más, trasladado al "mundo como hecho". Aparte de que Stenius no dice si concibe los predicados como 'universalia ante rem', 'in re' o 'post rem' y de que aceptemos o no el concepto de 'mundo como hecho' como equivalente legítimo de la concepción wittgensteiniana del 'mundo como totalidad de los hechos'; hay que objetar al procedimiento de Stenius que no ha dado razón o justificación alguna para pasar del modelo analógico al supuesto modelo del Tractatus, ni ha justificado tampoco el establecimiento de la analogía, la cual resulta asi arbitraria.

Pero además hay pasajes en el Tractatus que llevan a rechazar esta interpretación, incluso desde el punto de vista textual. Un pasaje decisivo en este sentido es:

4.24 Die Namen sind die einfachen Symbole, ich deute sie durch einzelne Buchstaben ("x", " $y$ ", " $z$ ") an. Den Elementarsatz schreibe ich als Funktion der Namen in der Form: " $f x ", ~ " \Phi(x, y)$ ", etc.

Oder ich deute ihn durch die Buchstaben $p, q$, $r$ an.

el cual interpreta Stenius de la siguiente manera:

"Elementary sentences may symbolically be written in the form $\mathrm{fx}$ or $\Phi(\mathrm{x}, \mathrm{y})$ (or $\mathrm{xRy}$ ) etc.... When Wittgenstein says that sentences of this form consist of names he obviously means not only that letters like ' $x$ ' or ' $y$ ' are names of individual objects but also that the predicate letters ' $f$ ' or ' $\Phi$ ' or ' $R$ ' are names of predicates." '12

Pero si decimos que una proposición elemental puede ser representada mediante $\Phi(\mathrm{x}, \mathrm{y})$, y decimos además que tanto $\mathrm{x}$, como $\mathrm{y}$, como $\Phi$ son nombres; entonces, de acuerdo con $4.22,3.21,3.22,2.13,2.13], 2.15, \mathrm{x}, \mathrm{y}, \Phi$ representan los

12 Stenius, op. cit., p. 126. 
objetos del hecho atómico y sus relaciones, las relaciones de los objetos. Esto es, además de $\Phi$, que relaciona $\mathrm{x}$ e $\mathrm{y}$, es necesaria una nueva relación, digamos $\theta$, que relacione estos tres 'objetos'. Así tendremos una nueva proposición elemental $\boldsymbol{\theta}(\Phi(\mathrm{x}, \mathrm{y}))$. Pero ahora será necesaria una nueva relación y así 'ad infinitum'.

El segundo argumento de Stenius pretende que si decimos, de acuerdo con 2.01, que un predicado 'une' (verbindet) objetos particulares para formar un hecho atómico, esto tendrá sentido mientras se trate de predicados multiargumentales (relaciones), pero al tratar con predicados uniargumentales (propiedades), como v.gr., 'A es rojo', habría que decir que el predicado 'rojo' une al objeto particular ' $A$ ' para formar un hecho atómico, donde el término 'unir' no tendria sentido. Por lo que, si hemos de consi. derar el hecho atómico como una unión (Verbindung) de objetos, tendremos que considerar el predicado 'rojo', esto es, la propiedad de ser rojo, como un objeto.

Contra este argumento se puede decir que la proposición 'A es rojo' no es elemental, según 6.3751; además de que Wittgenstein no habla en 2.01 de que los objetos sean unidos en el hecho atómico por un nuevo tipo de entidad, sim. plemente dice que el hecho atómico es una unión de objetos.

\section{VII}

La interpretación del concepto de objeto que mejor se adapta al Tractatus, esto es, la que puede ser aplicada a mayor número de proposiciones de éste sin provocar contradicciones, es la llamada interpretación nominalista. Es también la que cuenta con mayor número de adeptos entre los autores que se han ocupado específicamente del problema de los objetos. Una de las mejores exposiciones de esta interpretación es la de Copi, ${ }^{13}$ sobre la cual han trabajado otros

13 Copi, Objects, properties and relations in the Tractatus (en Copi, op. cit., pp. 167-186). Anterior a Copi en la interpretación nominalista es la citada obra de Anscombe. 
autores, modificándola en algunos puntos para hacerla más coherente con el contexto.

Copi parte del supuesto de que el concepto wittgensteiniano de objeto debe ser equivalente a una o más de las categorías metafísicas tradicionales de particular, propiedad y relación.

El primer paso de Copi es considerar la posibilidad de que entre los objetos del Tractatus puedan incluirse relaciones. Tal posibilidad es excluida por dar origen a una regresión 'ad infinitum' que vicia el concepto de objeto. Se trata de lo siguiente: si analizamos un hecho atómico en objetos individuales y relaciones, donde las relaciones sirven para 'unir' los objetos en el hecho atómico, y damos a las relaciones el carácter de objetos, será necesaria una nueva relación que una las relaciones anteriores y los objetos individuales, y así sucesivamente. Tratar de solventar el problema estipulando una diferencia categorial entre objetos y relaciones que no requiera nada para unirlos resulta ciertamente 'ad hoc'. Además, aparte del argumento general, creemos haber demostrado, de acuerdo con Copi, y sobre base textual, que tal regresión se produciría en el Tractatus si se intenta incluir relaciones entre sus objetos.

El segundo paso de Copi es considerar si las propiedades pueden ser consideradas objetos. Tal posibilidad es recha. zada en base a los siguientes argumentos: en primer lugar, el uso del término 'objeto' referido a propiedades en 4.123 es despreciado en la frase inmediata siguiente. En segundo lugar, el axioma de reducibilidad ${ }^{14}$ afirma la existencia de propiedades, y Wittgenstein habla de imaginar mundos en los que el axioma no es válido (6.1233), lo cual implicaría que las propiedades no han de ser las mismas en todo mundo imaginado. Pero, puesto que los objetos son los mismos en todo mundo imaginable $(2.022 ; 2.023)$, se sigue que los objetos no incluyen propiedades. En tercer lugar habla Copi de una prueba simbólica, entendiendo por

14 Russell, Principia Mathematica + 12.1 . 
tal cosa el hecho de que en el Tractatus los objetos sean representados en la forma en que usualmente se representan individuos y no propiedades $(4.1211 ; 4.1272 ; 4.24)$. En cuarto lugar, el Tractatus distingue entre propiedades materiales y formales. Los objetos no pueden ser propiedades formales, pues éstos pueden ser representados $(3.22 ; 3.221$; $3.203 ; 4.0312$ ), mientras aquellas no pueden serlo (4.12; 4.121 ; 4.0312). Tampoco pueden ser los objetos propiedades materiales, pues 2.0231 afirma que las propiedades materiales son "... erst durch die Konfiguration der Gegenstände gebildet", lo cual conducirá a un círculo vicioso si se pretendiera que las propiedades materiales son objetos. En quinto lugar, según 2.02 y 2.021 , los objetos son simples y no pueden ser compuestos. Siendo algunas propiedades complejas, no podrán ser consideradas como objetos. Lo más que se podrá pretender es que algunas propiedades, las propiedades simples, son objetos. Pero las propiedades más simples que se conocen, v.gr., los colores, no pueden ser objetos $(4.211 ; 6.3751)$.

Establecido que los objetos del Tractatus no pueden ser ni propiedades ni relaciones, Copi intenta establecer qué tipo de particulares han de ser dichos objetos. Para ello establece una distinción entre: 1) particulares con propiedades formales y materiales, 2) particulares desnudos, con propiedades formales, pero sin propiedades materiales, y 3) particulares absolutamente desnudos, sin propiedades. Aceptando que hay en el Tractatus evidencias para pensar que los objetos tienen propiedades materiales, Copi se inclina, sin embargo, a interpretarlos como particulares desnudos, en base a los siguientes argumentos: en primer lugar, Copi interpreta 2.0232 como una afirmación sinecdóquica de que los objetos no poseen propiedades materiales. En segundo lugar, los hechos pueden ser descritos $(3.144 ; 3.24 ; 4.023)$, mientras que los objetos sólo pueden ser nombrados (3.221). Si un objeto tuviera una propiedad material, ello constituiría un hecho cuya afirmación vendría a ser una descripción del objeto, contra lo establecido. Por último, dado 
que los signos proposicionales son analizables (3.25), no siéndolo los nombres (3.26), todo signo proposicional debe constar por lo menos de dos elementos; y como las proposiciones elementales deben tener la misma multiplicidad matemática que el hecho atómico que afirman (4.04), todo hecho atómico debe tener por lo menos dos elementos. Estando los hechos atómicos constituidos por objetos (2.01; 2.0272), todo hecho atómico debe estar constituido por lo menos por dos objetos. De tal manera, si un objeto tuviera una propiedad material, ello constituiría un hecho atómico con un solo objeto, contra lo establecido.

La primera parte de la tesis de Copi, la interpretación de los objetos como particulares y no como predicados, es aceptada por Anscombe, Griffin, Pitcher, Keyt y Sellars, entre otros; la segunda parte de la tesis, la caracterización de los objetos como particulares desnudos, es, por el contrario, generalmente impugnada, aunque no siempre en base a los mismos elementos. Los que presentamos nos parecen suficientes.

En primer lugar la evidencia textual sobre las propiedades materiales de los objetos, que Copi desprecia conscientemente, es bastante importante:

2.01231 Um einen Gegenstand zu kennen, muss ich zwar nicht seine externen - aber ich muss alle seine internen Eigenschaften kennen.

2.0233 Zwei Gegenstände von der gleichen logischen Form sind - abgesehen von ihren externen Eigenschaften - von einander nur dadurch unterschieden, dass sie verschieden sind.

4.023 (...) Wie die Beschreibung einen Gegenstand nach seinen externen Eigenschaften, so beschreibt der Satz die Wirklichkeit nach ihren internen Eigenschaften. (...)

$4.122 \quad(.$.$) Ich führe diese Ausdrücke ein, um der$ Grund der, bei den Philosophen sehr verbreitete Verwechslung zwischen den internen Re- 
lationen und den eigentlichen (externen) Relationen zu zeigen. (...)

El segundo y tercer argumentos de Copi pierden validez al dar una explicación, coherente dentro de la tesis nominalista, del hecho de que un objeto tenga propiedades materiales.

El problema a resolver consiste en que Wittgenstein diga que una proposición puede ser escrita simbólicamente como ' $\mathrm{fa}$ ', ${ }^{15}$ cuando habíamos llegado a la conclusión de que ' $\mathrm{f}$ en ' $\mathrm{fa}$ ' no puede ser un nombre, no pudiendo tampoco ser un símbolo para una propiedad o relación. La explicación de Griffin procede de la siguiente manera: supongamos que ' $a$ ', ' $b$ ', 'c', ' $d$ ', etc., son nombres; entonces podríamos escribir la proposición elemental 'a-b-c-d' constituida sólo por nombres. Ahora bien, esta proposición es función de las expresiones contenidas en ella (3.318) -entendiendo por expresión las partes de una proposición que caracterizan su sentido (3.31) - . Si se quisiera poner de relieve alguna expresión contenida en nuestra proposición elemental, podríamos hacerlo convirtiendo uno o más de los nombres que aparecen en ella en variables y dejando el resto cons. tante, v.gr., ' $x$-b-c-d'. Esto sería una expresión contenida en la proposición elemental y puede abreviarse escribiendo ' $f x$ ', de donde, sustituyendo de nuevo ' $a$ ', obtenemos ' $f a$ '. Así resulta que ' $f$ ' no es propiamente un nombre, pero puede ser vista como una abreviatura permisible de una configuración de nombres, pudiendo 'fa' ser llamada una proposición elemental.

Esta explicación puede ser trasladada 'mutatis mutandis' al hecho de que un objeto tenga una propiedad material, conservando el principio de la interpretación nominalista. Basta pensar en el hecho atómico representado por la proposición elemental 'a-b-c-d' y considerar en él el objeto

15 Wittgenstein utiliza ' $x$ ', etc., como constantes. Nosotros emplearemos, siguiendo a Griffin (op. cit., pp. $57 \mathrm{n}$ ) ' $a$ ', 'b', 'c', etc., de acuerdo con el uso establecido, $y$ dejaremos ' $x$ ', etc. para designar variables. 
designado por ' $a$ ', el cual está configurado con los otros objetos, los cuales, a su vez, están configurados entre sí de una manera particular. Nada impide que consideremos la particular configuración de los objetos designados por ' $b$ ', 'c' y 'd', mientras no sea analizada como una propiedad material. Se podría objetar entonces que ' $f$ ' en ' $f a$ ' sería un símbolo para una propiedad en una proposición elemental. Objeción que quedaría eliminada al responder que ' $\mathrm{fa}$ ' no tiene carácter de proposición elemental mientras ' $\mathrm{f}$ ' no está completamente analizada, lo cual no impide que utilicemos ' $f$ ' como forma abreviada de representar la configuración, completamente analizada '-b-c-d'.

Por último, esto permitiría explicar satisfactoriamente 2.0232: los objetos aislados no tienen propiedades materiales, pues éstas surgen en las configuraciones, lo cual en forma alguna quiere decir que a un objeto no se le pueda atribuir una propiedad material.

Aparte de los usos atípicos del término 'objeto' y de las proposiciones $2.0131,3.1431,4.123,5.02$, donde el uso del término contradice el contexto definitorio del mismo, las otras proposiciones del Tractatus donde aparece pueden ser satisfactorialmente interpretadas en base a la interpretación nominalista. Esta interpretación cumple mejor que ninguna otra la condición de coherencia con lo que hemos llamado contexto definitorio. Pero el precio que paga por ello es alto. Se trata, como observa Allaire, ${ }^{16}$ de una interpretación eminentemente verbal. Se ha logrado dar otro nombre a lo que el Tractatus llama objetos, y se ha logrado dar la impresión de que esto está relacionado con conceptos filosóficos tradicionales. Hemos dicho "dar la impresión", pues los particulares del Tractatus no son en forma alguna los particulares de la tradición filosófica. Los particulares del Tractatus son simples, sin que sepamos cuál es el criterio de simplicidad que los diferencia de otros particulares.

16 Allaire, The Tractatus: Nominalistic or Realistic? (in: Copi, op. cit., pp. $325-341$ ). 
Por decirlo así, la interpretación nominalista es muy útil para nada: seguimos sin saber cómo hemos de interpretar los objetos del Tractatus; y creemos haber demostrado que de acuerdo al sistema expuesto en la obra es necesario saberlo para que dicho sistema tenga realmente carácter de tal y pueda ser utilizado como explicación del lenguaje y de su relación con una realidad extra-lingüística. Mientras ignoremos cuál es la naturaleza de los objetos del Tractatus, sólo podremos considerar a la obra como un conjunto de notas sobre posibles hipótesis para estudiar el lenguaje. ${ }^{17}$ Creemos que el concepto de objeto del Tractatus no admite una interpretación que resuelva satisfactoriamente los problemas que el sistema quiere resolver, y que sea además compatible con las proposiciones más importantes del contexto definitorio. Demostrar o intentar demostrar totalmente esta hipótesis, si es que ello es posible, representaría una empresa demasiado larga y compleja para un punto de partida tan negativo. Nadie demuestra totalmente la falsedad de lo que rechaza ... y no seremos la excepción. Nos parece, sin embargo, que la discusión realizada es una prueba razonable de lo que decimos. Y podríamos agregar, como hace Favrholdt, unos cuantos problemas no tratados que dificultarían aún más cualquier intento de resolver la cuestión de los objetos, como, por ejemplo, la existencia de objetos síquicos $(2.1 ; 2.141 ; 3.301 ; 4.04)$ o el hecho de que los nombres deban ser objetos $(2.1 ; 2.141 ; 4.01 ; 4.04)$.

17 Se entiende que nos referimos aquí a la parte de la obra que quiere ser un sistema del lenguaje. 
I. According to the Tractatus the world is made up of the whole body of facts. Some of these are used in order to convey other facts. Language, composed of the whole body of propositions, is a collection of facts reproducing other facts. Every proposition can be constructed by starting from basic propositions. Basic propositions are the linguistic primaries. Basic propositions reproduce atomic facts. Atomic facts are combinations of objects. Objects combined in atomic facts become the final reference of language.

On the other hand, objects make up the substance of the world; they are what is unalterable and subsistent. Thus objects are the fundamental ontological elements and at the same time the ultimate reference of language. No study of the Tractatus which deals with the problem of language can proceed without first establishing a satisfactory notion of the concept 'object'.

In this article two questions are investigated: (1) What is the place of the concept 'object' in the Tractatus? and in particular is it or is it not an assumption within the system developed in the Tractatus? and (2) How must the concept 'object' be interpreted?

II. The only passages in which the need for the concept 'object' is stated are 2.021 to 2.0211. Their general meaning is as follows: If we were to deny the existence of objects, then propositions would lose their elementary character, and both their sense and their truth value would have to be referred to new propositions. The sense of any given propositions would thus be lost in a 'regressus ad infinitum'. But as there are propositions with sense it is essential to accept the existence of objects. The passage has the form of a 'reductio ad absurdum'. Reduced to its simplest form it would read: Propositions are classified into elementary and nonelementary ones. The sense of non elementary propositions depends on the sense of elementary propositions and the sense of the latter depends on the existence of objects. Hence the sense of any proposition depends in the last resort on the existence of objects. If we deny the existence of objects we must deny the existence of meaningful propositions. Since meaningful propositions do exist we must assume the existence of objetcs. If our interpretation is a correct one the existence of objects is an assumption, because if we consider the reduction as a demonstration we must conclude that it is a false one. Which is the answer to our first question. 
III. As regards the second question, two tendencies are noticeable among the interpreters of the Tractatus: one that defends the thesis that the language referred to in the work is, in the last resort, ordinary language, while the other defends the thesis that the language referred to in the Tractatus is not an historically given language but an ideal language towards which any given language tends, or should tend. One of the most radical representatives of this latter thesis is Max Black. He holds that the semantics of the Tractatus "is that of a lingua abscondita grounded in 'elementary propositions' whose existence is guaranteed only by metaphysical inference. We can produce no elementary propositions and would not recognize them if we had them."

Against this interpretation it might be argued that the nonelementary propositions referred to by Wittgenstein are those of ordinary language, as is demonstrated in many passages. If elementary propositions were unknowable the meaning and truth value of non-elementary propositions could be determined without reference to them, which contradicts Wittgenstein's thesis, or it would be absolutely impossible to determine them. Hence, either elementary propositions are knowable, or language can not communicate any meaning at all.

IV. Nor can the concept of 'object' mean either 'thing' (in the ordinary meaning of the word) or ontological entities, which are interpretations mentioned by, among others, A. Maslow. The word 'object' can not be taken to mean 'things', as the latter are neither simple, nor unalterable nor subsistent, as in the former. Nor can 'object' be used to refer to the final ontological entities of a 'world in itself'. Because if by 'world in itself' we understand 'a world completely independent of the knowledge that we have about it', there is no mention of this, nor is this problem dealt with in the Tractatus. Furthermore, the limits of the world are identical with those of language, and what is outside those limits is said in the "Prologue" to be 'nonsense'.

V. Favrholdt considers the interpretation of the concept of object as sense-data, based on 2.013 and 2.0131. Although he feels that Wittgenstein rejects this interpretation in other passages, he insists that the Tractatus leads up to it. Neverheless, two arguments can be adduced against this: (1) Supposing that simple sense-data could be determined, they would be changing and unstable, which is contrary to what Wittgenstein says about objects. (2) The physical propositions that analyze such simples would fall outside language, which is contrary to what is said in 6.3431. In fact 
such propositions can not be elementary propositions because they do not refer to configurations of objects, and they can not be truth functions of elementary propositions because (according to the interpretation in question) the latter would be propositions about complexes, since simple sense-data are physically analyzable.

VI. By objects, E. Stenius understands particulars, properties and relations. But this interpretation is based on arguments whose validity is very doubtful. The exegesis is based on the construction of a heuristic analogy between Wittgenstein's 'field of perception' and his 'world as a fact'. The analysis of the field of perceptivn, which can be analyzed into particulars and predicates, is transferred to the world as a fact, without giving any reason for such a step or justifying the analogy. Furthermore, there are passages in the Tractatus that lead us to reject this interpretation. For example, 4.23 can not be interpreted, as Stenius does i.e., meaning that in the elementary proposition ' $\Phi(x, y)$ ', both ' $x$ ' and ' $y$ ' and the sign of relation ' $\Phi$ ' are names, and therefore represent objects. If in fact ' $\Phi$ ' represents an object a new relation would be required to relate these three objects $(\mathrm{x}, \mathrm{y}$ and $\Phi)$; we should then have a new proposition: $\theta(\Phi(\mathrm{x}, \mathrm{y}))$, and so on ad infinitum.

VII. The interpretation of the concept of object that is best adapted to the Tractatus and enjoys the greatest measure of support is the so-called nominalistic interpretation. One of the best explanations of it is given by Copi. Copi starts with the supposition that the concept 'object' must be equivalent to one or more of the traditional metaphysical categories: particular, property and relations. By means of various arguments he establishes that the objects of the Tractatus can be neither properties nor relations. He then tries to establish what kind of particulars they must be. Admitting that there is evidence in the Tractatus that objects have material properties, Copi is nevertheless inclined to interpret them as mere particulars without properties, on the basis of a great deal of argument. While the interpretation of objects as particulars is accepted by many authors, their characterization as mere particulars is generally contested.

In the first place, the textual evidence regarding material properties of the object is of considerable importance. In addition, the problem to be solved in the nominalistic interpretation is that Wittgenstein says that a proposition may be symbolized by ' $f a$ ', whereas we have reached the conclusion that ' $f$ ' in ' $f a$ ' can be neither a name, nor a symbol of a property nor a symbol of a relation. Griffin's interpretation is that the elementary proposition 
might be written as 'a-b-c-d', made up of nothing but names, and that ' $\mathrm{f}$ ' in ' $\mathrm{fa}$ ' may be regarded as an abbreviation of a configuration of names; this would designate a configuration of objects not yet analyzed (for example 'b-c-d') that is in relation with the object designated by ' $a$ '; in this way ' $\mathrm{fa}^{\prime}$ ' may be called an elementary proposition, since it designates a configuration of objects. This explanation may be applied to the fact that the object has a material property. We may consider the particular configuration of the objects designated by 'b', 'c', ' $d$ ' in the elementary proposition 'a-b-c-d' as a material property as long as it is not analyzed. We shall then use ' $\mathrm{f}$ ' as an abbreviation to represent the configuration 'b-c-d'. This would allow us to explain that, although isolated objects have no material properties, these may be attributed to them because they occur in configurations of objects.

Apart from a few atypical uses of the term 'object', the other propositions in which it appears in the Tractatus can be satisfactorily explained on the basis of the nomalistic interpretation, which is coherent with what we call defining context. But the price to be paid for this is very high. We are faced by an eminently verbal interpretation. A different name is given to what the Tractatus calls 'objects' and the impression is given that this is related to traditional philosophical concepts. The particulars established in the Tractatus are not in fact the particulars of philosophical tradition. The former are simple, and we have no means of knowing what test of simplicity differentiates them from other particulars. We still do not know how we are to interpret the 'objects' of the Tractatus, and under the system set forth in the work it is essential to know it. So long as we are ignorant of this the work can be no more than a collection of notes affording possible hypotheses for the study of language. We think that the concept of object in the Tractatus is not susceptible of an interpretation that might satisfactorily resolve the problems which the system is intented to resolve and that might furthermore be compatible with the most important propositions of the defining context. 